\title{
Status de la Société Internationale de Pathologie
}

\section{Géographique}

La Société Internationale de Pathologie Géographique se propose d'étu-dier les relations qui peuvent exister entre les maladies, notamment les lesions qui les caractérisent, et les milieux géographiques dans lesquels elles se déve-loppent.

Le siege social de la Société se trouve en Suisse.

Membres :

La Société recrute ses membres parmi les personnalités médicales et non médicales s'intéressant aux problèmes de pathologie géographique. Les candidatures doivent être adressées au Secretariat general.

Les membres de la Société se rencontrent tous les 3 ans en conference (§ 7); le cas échéant, ils y présentent des communications et participent aux débats scientifiques et administratifs. Ils ont le droit de vote au cours de la reunion de Tassemblée administrative de la Société.

Chaque membre est redevable d'une cotisation annuelle de fr. suisses 5.- qui sera versée au Secretaire general, soit directement, soit par Гintermédiaire des Comités nationaux avant le ler avril de chaque année. Le produit de ces cotisations est avant tout destine à couvrir les frais d'administration et de correspondance.

4. Comités régionaux et nationaux :

Dans chaque pays ou region géographique se constitue un Comité national ou regional, compose de personnalités médicales et non médicales. La constitution des Comités nationaux n'est pas nécessairement fixe ; elle peut être variable en fonction du sujet choisi pour une conference donnée. Ces Comités sont, en effet, charges des enquêtes et des recherches scientifiques décidées par la société et représentent ainsi des Commissions scientifiques de la Société. Chaque comité nommera un President et un Secretaire. Ce dernier assumera :

le contact avec le Secretaire general ;

il tiendra à jour une liste complete des membres pour son pays ; il se chargera, le cas échéant, de recueillir les cotisations des membres de son pays et du virement de ces cotisations au compte de la S.I.P.G. ;

il est charge de faire rédiger les rapports nationaux destines aux rapporteurs des conferences ( $\S$ 7).

5. La Société est administrée par un Comité directeur. Le Comité directeur comprend, de droit, le Secretaire general et les Presidents des deux conferences précédentes. III se compose, en outre, de six membres ordinaires, et de quatre membres adjoints. Ceux-ci sont élus, au cours de $\Gamma$ Assemblée administrative tenue à Toccasion de chaque conference, à la majorité relative des voix, par les membres de la Société presents à cette assemblée. Leur mandat est d'une 636 Statuts de la Société Internationale de Pathologie Géographique durée de trois ans, ou du moins reste valable jusqu'à la conference suivante. II est renouvelable. 
Une fois constitué, le Comité directeur désigne dans son sein un President et deux vicePrésidents, pour une durée de trois ans. Le President du Comité directeur est le President de la Société, et le President désigné de la conference suivante. III représente la Société avec le Secretaire general. Avec lui, il a la charge d'organiser la conference qu'il est appelé à présider. Son mandat n'est pas renouvelable à fin de celle-ci.

Le Secretariat general de la Société est une institution permanente qui assure les relations entre le Comité directeur et les Comités nationaux. Le Secretaire general gère les affaires de la Société en collaboration avec le Comité directeur, et plus particulièrement avec le President de ce Comité. II est charge de la correspondance générale de la Société et des relations avec les organisations Internationales auxquelles la Société est affiliée. II est charge de recueillir les documents provenant des différents pays, documents destines aux enquetes faites par la Société, il organise la partie scientifique des conferences en relation avec les Presidents locaux de ces Conferences, et assure la publication des comptes rendus de celles-ci en collaboration avec le Secretaire de la Conference. Le Secretaire general assure les functions de Trésorier de la Société.

La Société organise en general tous les 3 ans une conference. Le sujet de discussion est choisi si possible 3 ans à $\Gamma$ avance par ГAssemblée générale des membres, après consultation des Comités nationaux. Le Comité directeur choisit les rapporteurs nécessaires en collaboration avec les Comités nationaux. Le travail d'enquête, qui incombe aux Comités nationaux, est condense en un ou plusieurs rapports nationaux qui seront remis en temps voulu au Secretariat general par les soins des Secretaires des Comités nationaux dans les délais prévus ad hoc par le Secretaire general. Ce materiel forme la base des rapports présentés aux conferences par les personnalités choisies. II demeure la propriété intellectuelle des Comités nationaux. Les conferences sont réservées aux membres de la Société ; toutefois, toute personnalité s'intéressant aux questions de pathologie géographique ou plus particulièrement au sujet discuté, pourra y parti-ciper sous la responsabilité du Comité national de son pays.

Tout participant aux conferences verse une cotisation de conference, destinée à couvrir les frais occasionnés par la preparation et Torganisation de ces conferences, la publication des comptes rendus et les contributions obliga-toires aux organisations Internationales auxquelles la Société est affiliée. Le montant de ces cotisations est fixe par le Secretariat general et le President de la conference selon les exigences du moment et la valeur monétaire des différents pays. Les participants aux conferences ont droit à un exemplaire du volume des comptes rendus de la conference à laquelle ils ont assisté.

Les fonds de la Société sont gérés par le Secretaire general. Au cours de la seance administrative, prévue à Гoccasion de chaque conference, la Société nomme deux membres qui fonctionneront comme Commissaires aux comptes ; sur leur proposition, la Société se prononcera sur la décharge à accorder au Trésorier.

Statuts de la Société Internationale de Pathologie Géographique 637

10. Assemblée administrative :

$\mathrm{Au}$ cours de chaque conference, les membres presents se réuniront afin de délibérer sur les affaires internes de la Société. Ils procéderont en particulier : à la nomination du Comité directeur et à la revision des comptes. Ils discuteront et choisiront à la majorité des voix le prochain sujet d'enquête ainsi que le lieu de reunion de la prochaine conference. Tout membre de la Société a la faculté de formuler toute proposition qui lui paraît utile pour la bonne marche de la Société, à condition d'en informer le Secretaire general au moins un mois avant le debut de la conference. 
L'organisation pratique des conferences est déléguée au Comité national du pays où siégera la conference, et plus particulièrement au President de ce Comité national. C'est lui qui assurera l'organisation matérielle de la conference, qui se chargera, en relation avec le Secretaire general, de la redaction, de $\Gamma$ impression et de $\Gamma$ expédition des invitations et des programmes, de $\Gamma$ annonce de la conference à la presse, etc.

Après que les Comités nationaux en aient été prévenus au moins trois mois à $\Gamma$ avance, les presents Statuts pourront être modifies. Toute modification sera effectuée à la majorité de voix des Presidents des Comités nationaux ou de leurs représentants, presents à la Seance administrative qui se tiendra au cours d'une conference.

Juillet 1957. 